\title{
Control of Bifurcation in Internet Model with Time-Delay
}

\author{
Mohammad Darvishi", Haji Mohammad Mohammadinejad \\ Department of Mathematics, Faculty of Mathematics and Statistics, University of Birjand, Birjand, Iran \\ Email address: \\ m.darvishi@birjand.ac.ir (M. Darvishi),hmohammadin@birjand.ac.ir(H. M. Mohammadinejad) \\ *Corresponding author
}

To cite this article:

Mohammad Darvishi, Haji Mohammad Mohammadinejad. Control of Bifurcation in Internet Model with Time-Delay. Applied and Computational Mathematics. Vol. 5, No. 5, 2016, pp. 186-192. doi: 10.11648/j.acm.20160505.11

Received: September 1, 2016; Accepted: September 13, 2016; Published: October 8, 2016

\begin{abstract}
In this paper we study the Hopf bifurcation control problem of a Internet Model with Time-delay. The stable region and stability condition of the congestion control model are given by use of the linear stability analysis. When the system gain passes through a critical value, the system loses the stability and Hopf bifurcation occurs. Considering the negative influence caused by Hopf bifurcation, we apply Proportional - Derivative (PD) controller to postpone the onset of undesirable Hopf bifurcation. Numerical simulation results and figures confirm that the control strategy is efficient in controlling the Hopf bifurcation.
\end{abstract}

Keywords: Hopf Bifurcation, Bifurcation Control, PD Controller

\section{Introduction}

Congestion control is a topic that has been dealt with for a long time, and it has also become a facet of daily life for Internet users. Network congestion in data networking and queueing theory is the reduced quality of service that occurs when a network node is carrying more data than it can handle. Typical effects include queueing delay, packet loss or the blocking of new connections. A consequence of the latter two effects is that an incremental increase in offered load leads either only to a small increase or even a decrease in network throughput [4].

FAST TCP is a TCP congestion avoidance algorithm especially targeted at long-distance for high speed networks with large bandwidth-delay products and uses the queuing delay as congestion measure, which enables FAST TCP to detect congestion without packet loss [5]. Many research efforts have been devoted to the analysis of the stability property of FAST TCP. Wang and his colleagues have introduced a new discrete-time link model that fully captures the effect of self-clocking and compared it with the traditional continuous-time model and Showed that FAST TCP is globally stable on a single link in the absence of feedback delay [8]. In [6, 10, 13], authors have studied global asymptotically stability of FAST TCP based on a continue model. they prove that the system is always asymptotically stable for general networks in the absence of feedback delay. Zhang and Liu have proved that FAST TCP is locally asymptotically stable in the homogeneous networks on condition that the round trip propagation delay is smaller than the queuing delay [7]. FAST TCP network is in a structure of feedback inteconnection of two input-to-state stable systems, and have proved that the composite total system is also input-to-state stable and globally asymptotically stable by applying the Razumikhin type nonlinear small gain theorem. This result shows that FAST TCP is inherently globally asymptotically stable without any constraint on the tuning parameter a or update gain b [9].

By using communication delay as a bifurcation parameter in a fair dual algorithm of an Internet congestion control system, was shown that when the communication delay of the system passes through a critical value, a Hopf bifurcation occurs in such a dual model of one-order time-delay congestion control system [2]. The author in [12] has proposed $a$ state feedback method to control the bifurcation for a novel congestion control model. In [11], an impulsive control strategy has been applied to the FAST TCP and RED model for controlling bifurcation. And paper [3] has proposed a PD controller to controll the Hopf bifurcation in a complex 
networks model. Proportional-Integral-Derivative (PID) controller is a widely used control method for dynamics control in nonlinear system for its superior performance. A PID-controller consists of a PI control portion connected in serial with a PD control portion. PI control can improve the steady-state error at the expense of an increasing response time while PD control can improve the speed of response of a control system. In this paper, PD controller applied to FAST TCP and RED model, for postponing the occurrence of Hopf bifurcation. The PD control strategy can be applied to any component of a several-dimensional dynamical system and is still effective even when the system becomes chaotic.

The organization of this paper is as follows. Some results in uncontrolled system are concluded in Section 2. In Section 3, we study the Hopf bifurcation of Control system. In the next section, by the normal form method and the center manifold theory introduced by Hassard et al. [1], the direction of Hopf bifurcation and the stability of bifurcating periodic solutions are determined. In addition, the main results illustrated by examples with numerical simulations.

\section{Existence Hopf Bifurcation in Uncontrol FAST TCP Model}

The model can be described by the following nonlinear differential equations:

$$
\left\{\begin{array}{c}
\dot{x}(t)=a\left(\frac{b}{d+y\left(t-\tau_{2}\right)}-\frac{y\left(t-\tau_{2}\right) x(t)}{\left(d+y\left(t-\tau_{2}\right)\right)^{2}}\right) \\
\dot{y}(t)=\frac{1}{c}\left(\frac{x\left(t-\tau_{1}\right)}{d+y\left(t-\tau_{1}-\tau_{2}\right)}-c\right)
\end{array}\right.
$$

Where

$\mathrm{x}(\mathrm{t})$ : denotes congestion window of the source,

$\mathrm{c}$ : the transmission capacity,

$\mathrm{y}(\mathrm{t})$ : the queuing delay,

$\tau_{1}$ : the forward delay from source to link,

$\tau_{2}$ : the backward delay in the feedback path from link to source,

$\mathrm{d}$ : represents the constant round trip propagation time defined as the minimum achievable round trip delay.

$b(b>0)$ : the number of the packets that each source attempts to maintain in the network buffers at equilibrium point,

a: the source control parameter with $a \in(0,1]$.

The congestion window $\mathrm{x}(\mathrm{t})$ and the queuing delay $\mathrm{y}(\mathrm{t})$ are nonnegative. Let $\tau=\tau_{1}+\tau_{2}$ equation (1) can bewritten

$$
\left\{\begin{array}{c}
\dot{x}(t)=a\left(\frac{b}{d+y(t-\tau)}-\frac{y(t-\tau) x(t)}{(d+y(t-\tau))^{2}}\right) \\
\dot{y}(t)=\frac{1}{c}\left(\frac{x(t)}{d+y(t-\tau)}-c\right)
\end{array}\right.
$$

And the equilibrium point is $\left(x_{0}, y_{0}\right)=\left(d c+b, \frac{b}{c}\right)$.

With the linearizing system (2) about $\left(x_{0}, y_{0}\right)$ and transfer critical point of origin we have:

$$
\left\{\begin{array}{c}
\dot{x}(t)=-a b c \alpha^{2} x(t)-c^{3} d a \alpha^{2} y(t-\tau) \\
\dot{y}(t)=\alpha x(t)-c \alpha y(t-\tau)
\end{array}\right.
$$

Where $\alpha=\frac{1}{\mathrm{dc}+\mathrm{b}}$ and the characteristic equation (3) is

$$
\lambda^{2}+a b c \alpha^{2} \lambda+\left(a b c^{2} \alpha^{3}+c \alpha \lambda+c^{3} d a \alpha^{3}\right) e^{-\lambda \tau}=0
$$

We assume $\lambda=i \omega$ is a purely imaginary root of (4), then we can obtained

$$
\omega^{4}+\left(a^{2} b^{2} c^{2} \alpha^{4}-\alpha^{2} c^{2}\right) \omega^{2}-\left[a b c^{2} \alpha^{3}+c^{3} d a \alpha^{3}\right]^{2}=0
$$

So the equation (4) has a solution $\lambda=i \omega_{0}$ that $\omega_{0}>0$. Let $\lambda_{k}(\tau)=R_{k}(\tau)+i I_{k}(\tau)$ denote a root of equation (4) such that $\lambda_{k}\left(\tau_{k}\right)=i \omega_{0}$. Then

$$
\begin{gathered}
\tau_{\mathrm{k}}=\frac{1}{\omega_{0}}\left(\operatorname{Arcsin}\left[\frac{\alpha \mathrm{c} \omega_{0}^{3}+\mathrm{a}^{2} \mathrm{~b}^{2} \mathrm{c}^{3} \alpha^{5} \omega_{0}+\mathrm{a}^{2} \mathrm{bc} \mathrm{c}^{4} \mathrm{~d} \alpha^{5} \omega_{0}}{\left(\mathrm{abc}{ }^{2} \alpha^{3}+\mathrm{c}^{3} \mathrm{da} \alpha^{3}\right)^{2}+\alpha^{2} \mathrm{c}^{2} \omega_{0}^{2}}\right]+2 k \pi\right) \\
k=0,1,2, \ldots
\end{gathered}
$$

We still need to check the following transversal condition in order to verify the onset of Hopf bifurcation.

Lemma 1. In the equation of (4)

$$
\left.\operatorname{Re}\left(\frac{\mathrm{d} \lambda}{\mathrm{d} \tau}\right)\right|_{\tau=\tau_{\mathrm{k}}}>0
$$

Proof. Let $A=c \alpha, B=a b \alpha$ and $C=a d$ then

$$
\begin{gathered}
\frac{d \lambda}{d \tau}=\frac{\left(A^{2} B \lambda+A \lambda^{2}+A^{3} C \lambda\right) e^{-\lambda \tau}}{2 \lambda+A B+\left(-A^{2} B \tau+A-A \lambda \tau-A^{3} C \tau\right) e^{-\lambda \tau}} \\
\left.\operatorname{Re}\left(\frac{d \lambda}{d \tau}\right)\right|_{\tau=\tau_{k}}=\frac{M_{1}+M_{2}+M_{3}}{M_{4}}
\end{gathered}
$$

That

$$
\begin{gathered}
\mathrm{M}_{1}=\left[2 \mathrm{~A} \omega_{0}^{2}+\mathrm{A}^{3} \mathrm{~B}^{2} \omega_{0}+\mathrm{A}^{4} \mathrm{BC} \omega_{0}\right] \sin \omega_{0} \tau_{\mathrm{k}} \\
M_{2}=\left[2 A^{2} B \omega_{0}^{2}+2 A^{3} C \omega_{0}^{3}-A^{2} B \omega_{0}\right] \cos \omega_{0} \tau_{k} \\
M_{3}=A^{2} B \tau_{k}+A^{3} C \tau_{k}-A-A^{2} B \tau_{k} \omega_{0}-A^{3} C \tau_{k} \omega_{0} \\
M_{4}=\left[A B \cos \omega_{0} \tau_{k}-2 \omega_{0} \sin \omega_{0} \tau_{k}-A^{2} B \tau_{k}\right. \\
\left.+A-A^{3} C \tau_{k}\right]^{2}+\left[A B \sin \omega_{0} \tau_{k} 2 \omega_{0} \cos \omega_{0} \tau_{k}-A \omega_{0} \tau_{k}\right]^{2}
\end{gathered}
$$

Thus, according to

$$
\left\{\begin{array}{c}
\cos \omega_{0} \tau_{k}=\frac{\omega_{0}^{2}\left[\mathrm{~A}^{2} \mathrm{~B}+\mathrm{A}^{3} \mathrm{C}\right]-\mathrm{A}^{2} \mathrm{~B} \omega_{0}^{2}}{\left[\mathrm{~A}^{2} \mathrm{~B}+\mathrm{A}^{3} \mathrm{C}\right]^{2}+\mathrm{A}^{2} \omega_{0}^{2}} \\
\sin \omega_{0} \tau_{\mathrm{k}}=\frac{\mathrm{AB} \omega_{0}\left[\mathrm{~A}^{2} \mathrm{~B}+\mathrm{A}^{3} \mathrm{C}\right]+\mathrm{A} \omega_{0}^{3}}{\left[\mathrm{~A}^{2} \mathrm{~B}+\mathrm{A}^{3} \mathrm{C}\right]^{2}+\mathrm{A}^{2} \omega_{0}^{2}} \\
2 \omega_{0}^{2}=\mathrm{A}^{2}-\mathrm{A}^{2} \mathrm{~B}^{2}+\sqrt{\left[\mathrm{A}^{2} \mathrm{~B}^{2}-\mathrm{A}^{2}\right]^{2}+4\left[\mathrm{~A}^{2} \mathrm{~B}+\mathrm{A}^{3} \mathrm{C}\right]^{2}}
\end{array}\right.
$$

The proof is completed.

Therefore, the transversal condition for Hopf bifurcation in the system (2) is satisfied. We conclude above analyzes in following Theorem.

Theorem 2. For the system (2), there exists a Hopf bifurcation emerging from its equilibrium $\left(x_{0}, y_{0}\right)$ when the positive parameter $\tau$ passes through the critical value $\tau_{k}$. 


\section{Hopf Bifurcation in Controlled Model Based on PD Controller}

In this section, we focus on designing a controller to control the Hopf bifurcation in model based on the PD control strategy. Apply the PD control to system (2) then:

$$
\left\{\begin{array}{l}
\dot{x}(t)=a\left(\frac{b}{d+y(t-\tau)}-\frac{y(t-\tau) x(t)}{(d+y(t-\tau))^{2}}\right)+k_{p}\left(x(t)-x_{0}\right)+ \\
\dot{y}(t)=\frac{1}{c}\left(\frac{x(t)}{d+y(t-\tau)}-c\right)+k_{p}\left(y(t)-y_{0}\right)+k_{d} \dot{y}(t)
\end{array}\right.
$$

Where

$k_{p}$ : proportional control parameter, $-1<k_{p}<1$

$k_{d}$ : derivative control parameter, $-1<k_{d}<1$

Let $p(t)=x(t)-x_{0}, q(t)=y(t)-y_{0}$. system (10) about the equilibrium point, we get

$$
\left\{\begin{array}{c}
\dot{\mathrm{p}}(\mathrm{t})=\frac{1}{1-\mathrm{k}_{\mathrm{d}}}\left[\left(\mathrm{k}_{\mathrm{p}}-\mathrm{abc} \alpha^{2}\right) \mathrm{p}(\mathrm{t})-\mathrm{c}^{3} \mathrm{da} \alpha^{2} \mathrm{q}(\mathrm{t}-\tau)\right] \\
\dot{\mathrm{q}}(\mathrm{t})=\frac{1}{1-\mathrm{k}_{\mathrm{d}}}\left[\alpha \mathrm{p}(\mathrm{t})+\left(\mathrm{k}_{\mathrm{p}}-\mathrm{c} \alpha\right) \mathrm{q}(\mathrm{t}-\tau)\right]
\end{array}\right.
$$

The characteristic equation of which is

$$
\lambda^{2}+\left(\frac{A B-k_{p}}{1-k_{d}}\right)+\left(\frac{\left(k_{p}-A B\right)\left(k_{p}-A\right)+A^{3} C}{\left(1-k_{d}\right)^{2}}+\frac{A-k_{p}}{1-k_{d}} \lambda\right) e^{-\lambda \tau}=0
$$

Let $\lambda=i \omega$ is root equation (12). So

$$
\omega^{4}+\left[\left(\frac{A B-k_{p}}{1-k_{d}}\right)^{2}-\left(\frac{A-k_{p}}{1-k_{d}}\right)^{2}\right] \omega^{2}-\left(\frac{\left(k_{p}-A B\right)\left(k_{p}-A\right)+A^{3} C}{\left(1-k_{d}\right)^{2}}\right)^{2}=0
$$

Then the equation (13) has a solution $\lambda=i \omega_{0}^{*}$ that $\omega_{0}^{*}>0$ and also

$$
\left\{\begin{array}{c}
2 \omega_{0}^{* 2}=M\left(k_{p}, k_{d}\right)+N\left(k_{p}, k_{d}\right) \\
\tau_{k}^{*}=\frac{1}{\omega_{0}^{*}}\left(\operatorname{Arcsin}\left(\frac{P\left(k_{p}, k_{d}\right)}{Q\left(k_{p}, k_{d}\right)}\right)+2 k \pi\right)
\end{array}\right.
$$

Where

$$
\left\{\begin{array}{c}
M\left(k_{p}, k_{d}\right)=\left(\frac{c \alpha-k_{p}}{1-k_{d}}\right)^{2}-\left(\frac{a b c \alpha^{2}-k_{p}}{1-k_{d}}\right)^{2} \\
N\left(k_{p}, k_{d}\right)=\sqrt{M^{2}\left(k_{p}, k_{d}\right)+4\left(\frac{\left(k_{p}-a b c \alpha^{2}\right)\left(k_{p}-c \alpha\right)+(c \alpha)^{3} a d}{\left(1-k_{d}\right)^{2}}\right)^{2}} \\
P\left(k_{p}, k_{d}\right)=\left(\frac{c \alpha-k_{p}}{1-k_{d}}\right) \omega_{0}^{* 3}+\left(\frac{a b c \alpha^{2}}{1-k_{d}}\right)\left(\frac{\left(k_{p}-a b c \alpha^{2}\right)\left(k_{p}-c \alpha\right)+(c \alpha)^{3} a d}{\left(1-k_{d}\right)^{2}}\right) \omega_{0}^{*} \\
Q\left(k_{p}, k_{d}\right)=\left(\frac{c \alpha-k_{p}}{1-k_{d}}\right) \omega_{0}^{* 2}+\left(\frac{\left(k_{p}-a b c \alpha^{2}\right)\left(k_{p}-c \alpha\right)+(c \alpha)^{3} a d}{\left(1-k_{d}\right)^{2}}\right)^{2}
\end{array}\right.
$$

According to what was described in section 2, the controlled system (10) undergoes Hopf bifurcation at $\left(x_{0}, y_{0}\right)$ when $\tau=\tau_{k}^{*}, k=0,1,2, \ldots$

\section{Stability and Direction of Bifurcating Periodic Solutions}

In this section, by using normal form theorem and center manifold theory, we analyze the direction and the stability of bifurcating periodic solutions when $\tau=\tau_{k}^{*}$ and present numerical simulations to verify theoretical analysis obtained in the previous sections.

Let $y_{1}(t)=x(t)-x_{0}, y_{2}(t)=y(t)-y_{0}$. The expansion of equation (10) about the equilibrium point is

$$
\left\{\begin{array}{c}
\dot{y}_{1}(t)=a_{1} y_{1}(t)+a_{2} y_{2}(t-\tau)+a_{3} y_{1}(t) y_{2}(t-\tau)+ \\
a_{4} y_{2}{ }^{2}(t-\tau)+a_{5} y_{1}(t) y_{2}{ }^{2}(t-\tau)+a_{6} y_{2}{ }^{3}(t-\tau)+\cdots \\
\dot{y}_{2}(t)=b_{1} y_{1}(t)+b_{2} y_{2}(t-\tau)+b_{3} y_{1}(t) y_{2}(t-\tau) \\
b_{4} y_{2}{ }^{2}(t-\tau)+b_{5} y_{1}(t) y_{2}{ }^{2}(t-\tau)+b_{6} y_{2}{ }^{3}(t-\tau)+\cdots
\end{array}\right.
$$

Where

$$
\left\{\begin{array}{c}
\mathrm{a}_{1}=\frac{\mathrm{k}_{\mathrm{p}}-\mathrm{abc} \alpha^{2}}{1-\mathrm{k}_{\mathrm{d}}}, \mathrm{a}_{2}=\frac{-\mathrm{ac} \mathrm{c}^{3} \mathrm{~d} \alpha^{2}}{1-\mathrm{k}_{\mathrm{d}}}, \mathrm{a}_{3}=\frac{\mathrm{a} \alpha^{3}\left(\mathrm{c}^{2} \mathrm{~b}-\mathrm{c}^{3} \mathrm{~d}\right)}{1-\mathrm{k}_{\mathrm{d}}} \\
\mathrm{a}_{4}=\frac{4 a c^{4} \mathrm{~d} \alpha^{3}}{1-\mathrm{k}_{\mathrm{d}}}, \mathrm{a}_{5}=\frac{\mathrm{a} \alpha^{4}\left(4 \mathrm{c}^{4} \mathrm{~d}-2 \mathrm{c}^{3} \mathrm{~b}\right)}{1-\mathrm{k}_{\mathrm{d}}}, \mathrm{a}_{6}=\frac{-18 \mathrm{ac} \mathrm{c}^{4} \mathrm{~d} \alpha^{4}}{1-\mathrm{k}_{\mathrm{d}}} \\
\mathrm{b}_{1}=\frac{\mathrm{k}_{\mathrm{p}}+\alpha}{1-\mathrm{k}_{\mathrm{d}}}, \mathrm{b}_{2}=\frac{-\mathrm{c} \alpha}{1-\mathrm{k}_{\mathrm{d}}}, \mathrm{b}_{3}=\frac{-\mathrm{c} \alpha^{2}}{1-\mathrm{k}_{\mathrm{d}}} \\
\mathrm{b}_{4}=\frac{2 \mathrm{c}^{2} \alpha^{2}}{1-\mathrm{k}_{\mathrm{d}}}, \mathrm{b}_{5}=\frac{2 \mathrm{c}^{2} \alpha^{3}}{1-\mathrm{k}_{\mathrm{d}}}, \mathrm{b}_{6}=\frac{-6 \mathrm{c}^{3} \alpha^{3}}{1-\mathrm{k}_{\mathrm{d}}}
\end{array}\right.
$$

According to what is described in [13] we define:

$$
\begin{aligned}
& \rho=\left(\begin{array}{c}
1 \\
\rho_{1}
\end{array}\right)=\left(\begin{array}{c}
1 \\
\frac{\mathrm{i} \omega_{0}^{*}-\mathrm{a}_{1}}{\mathrm{~b}_{1} \mathrm{e}^{-\mathrm{i} \omega_{0}^{*} \tau_{\mathrm{k}}^{*}}}
\end{array}\right), \rho^{*}=\left(\begin{array}{c}
\rho_{2} \\
1
\end{array}\right)=\left(\begin{array}{c}
\frac{-\mathrm{b}_{1}}{\mathrm{i} \omega_{0}^{*}+\mathrm{a}_{1}} \\
1
\end{array}\right) \\
& \mathrm{D}=\left[\left(\overline{\rho_{1}}+\rho_{2}\right)+\overline{\rho_{1}}\left(\rho_{2} \mathrm{a}_{2}+\mathrm{b}_{2}\right) \tau_{\mathrm{k}}^{*} \mathrm{e}^{-\mathrm{i} \omega_{0}^{*} \tau_{\mathrm{k}}^{*}}\right]^{-1} \\
& \mathrm{k}_{11}=\mathrm{a}_{3} \rho_{1} \mathrm{e}^{-\mathrm{i} \omega_{0}^{*} \tau_{\mathrm{k}}^{*}}+\mathrm{a}_{4} \rho_{1}{ }^{2} \mathrm{e}^{-2 \mathrm{i} \omega_{0}^{*} \tau_{\mathrm{k}}^{*}} \\
& \mathrm{k}_{12}=\mathrm{a}_{3}\left(\overline{\rho_{1}} \mathrm{e}^{-\mathrm{i} \omega_{0}^{*} \tau_{\mathrm{k}}^{*}}+\rho_{1} \mathrm{e}^{-\mathrm{i} \omega_{0}^{*} \tau_{\mathrm{k}}^{*}}\right)+2 \mathrm{a}_{4} \rho_{1} \overline{\rho_{1}} \\
& \mathrm{k}_{13}=\mathrm{a}_{3} \overline{\rho_{1}} \mathrm{e}^{\mathrm{i} \omega_{0}^{*} \tau_{\mathrm{k}}^{*}}+\mathrm{a}_{4}{\overline{\rho_{1}}}^{2} \mathrm{e}^{2 \mathrm{i} \omega_{0}^{*} \tau_{\mathrm{k}}^{*}} \\
& \mathrm{k}_{21}=\mathrm{b}_{3} \rho_{1} \mathrm{e}^{-\mathrm{i} \omega_{0}^{*} \tau_{\mathrm{k}}^{*}}+\mathrm{b}_{4} \rho_{1}{ }^{2} \mathrm{e}^{-2 \mathrm{i} \omega_{0}^{*} \tau_{\mathrm{k}}^{*}} \\
& \mathrm{k}_{22}=\mathrm{b}_{3}\left(\overline{\rho_{1}} \mathrm{e}^{\mathrm{i} \omega_{0}^{*} \tau_{\mathrm{k}}^{*}}+\rho_{1} \mathrm{e}^{\mathrm{i} \omega_{0}^{*} \tau_{\mathrm{k}}^{*}}\right)+2 \mathrm{~b}_{4} \rho_{1} \overline{\rho_{1}}
\end{aligned}
$$




$$
\begin{aligned}
\mathrm{k}_{23}=\mathrm{b}_{3} \overline{\rho_{1}} \mathrm{e}^{\mathrm{i} \omega_{0}^{*} \tau_{\mathrm{k}}^{*}}+\mathrm{b}_{4} \overline{\rho_{1}} \mathrm{e}^{2 \mathrm{i} \omega_{0}^{*} \tau_{\mathrm{k}}^{*}} \\
\mathrm{~g}_{20}=2 \overline{\mathrm{D}}\left(\overline{\rho_{2}} \mathrm{k}_{11}+\mathrm{k}_{21}\right) \\
\mathrm{g}_{11}=\overline{\mathrm{D}}\left(\overline{\rho_{2}} \mathrm{k}_{12}+\mathrm{k}_{22}\right) \\
\mathrm{g}_{20}=2 \overline{\mathrm{D}}\left(\overline{\rho_{2}} \mathrm{k}_{13}+\mathrm{k}_{23}\right) \\
\mathrm{E}_{1}=\left(\begin{array}{c}
\frac{\mathrm{b}_{2} \mathrm{k}_{12}-\mathrm{a}_{2} \mathrm{k}_{22}}{\mathrm{a}_{2} \mathrm{~b}_{1}-\mathrm{a}_{1} \mathrm{~b}_{2}} \\
\frac{\mathrm{a}_{1} \mathrm{k}_{22}-\mathrm{b}_{1} \mathrm{k}_{12}}{\mathrm{a}_{2} \mathrm{~b}_{1}-\mathrm{a}_{1} \mathrm{~b}_{2}}
\end{array}\right)
\end{aligned}
$$

$E_{2}=\left(\frac{\frac{\left(2 \mathrm{a}_{2} \mathrm{k}_{21}-2 \mathrm{~b}_{2} \mathrm{k}_{11}\right) \mathrm{e}^{-2 \mathrm{i} \omega_{0}^{*} \tau_{\mathrm{k}}^{*}}+4 \mathrm{ik}_{11} \omega_{0}^{*}}{\left(\mathrm{a}_{2} \mathrm{~b}_{2}-\mathrm{a}_{2} \mathrm{~b}_{1}-2 \mathrm{i} \mathrm{b}_{2} \omega_{0}^{*}\right) \mathrm{e}^{-2 \mathrm{i} \omega_{0}^{*} \tau_{\mathrm{k}}^{*}-2 \mathrm{ia}_{1} \omega_{0}^{*}-4 \omega_{0}^{* 2}}}}{\frac{2 \mathrm{~b}_{1} \mathrm{k}_{11}-2 \mathrm{a}_{1} \mathrm{k}_{21}+4 \mathrm{i} \mathrm{i}_{21} \omega_{0}^{*}}{\left(\mathrm{a}_{2} \mathrm{~b}_{2}-\mathrm{a}_{2} \mathrm{~b}_{1}-2 \mathrm{i}_{2} \omega_{0}^{*}\right) \mathrm{e}^{-2 \mathrm{i} \omega_{0}^{*} \tau_{\mathrm{k}}^{*}-2 \mathrm{ia}_{1} \omega_{0}^{*}-4 \omega_{0}^{* 2}}}}\right)$

$\left(\begin{array}{l}W_{1}^{(1)}(\theta) \\ W_{1}^{(2)}(\theta)\end{array}\right)=-\frac{i g_{11}}{\omega_{0}^{*}} \rho \mathrm{e}^{\mathrm{i} \omega_{0}^{*} \theta}+\frac{\mathrm{i} \overline{g_{11}}}{\omega_{0}^{*}} \bar{\rho} \mathrm{e}^{-\mathrm{i} \omega_{0}^{*} \theta}+\mathrm{E}_{1}$

$$
\left(\begin{array}{l}
\mathrm{W}_{2}^{(1)}(\theta) \\
\mathrm{W}_{2}^{(2)}(\theta)
\end{array}\right)=-\frac{\mathrm{ig}_{20}}{\omega_{0}^{*}} \rho \mathrm{e}^{\mathrm{i} \omega_{0}^{*} \theta}+\frac{\mathrm{i} \overline{g_{20}}}{3 \omega_{0}^{*}} \bar{\rho} \mathrm{e}^{-\mathrm{i} \omega_{0}^{*} \theta}+\mathrm{E}_{2} \mathrm{e}^{2 \mathrm{i} \omega_{0}^{*} \theta}
$$

$$
\begin{aligned}
\mathrm{k}_{14}= & \mathrm{a}_{3}\left[\frac{1}{2} \mathrm{~W}_{2}^{(1)}(0) \overline{\rho_{1}} \mathrm{e}^{\mathrm{i} \omega_{0}^{*} \tau_{\mathrm{k}}^{*}}+\mathrm{W}_{1}^{(1)}(0) \rho_{1} \mathrm{e}^{-\mathrm{i} \omega_{0}^{*} \tau_{\mathrm{k}}^{*}}+\frac{1}{2} \mathrm{~W}_{2}^{(2)}\left(-\tau_{\mathrm{k}}^{*}\right)+\mathrm{W}_{1}^{(2)}\left(-\tau_{\mathrm{k}}^{*}\right)\right]+\mathrm{a}_{4}\left[\mathrm{~W}_{2}^{(2)}\left(-\tau_{\mathrm{k}}^{*}\right) \overline{\rho_{1}} \mathrm{e}^{\mathrm{i} \omega_{0}^{*} \tau_{\mathrm{k}}^{*}}\right. \\
& \left.+2 W_{1}^{(2)}\left(-\tau_{k}^{*}\right) \rho_{1} e^{-i \omega_{0}^{*} \tau_{k}^{*}}\right]+a_{5}\left[\rho_{1}{ }^{2} e^{-2 i \omega_{0}^{*} \tau_{k}^{*}}+2 \rho_{1} \overline{\rho_{1}}+3 a_{6} \rho_{1}{ }^{2} \overline{\rho_{1}} e^{-i \omega_{0}^{*} \tau_{k}^{*}}\right. \\
\mathrm{k}_{24}= & \mathrm{b}_{3}\left[\frac{1}{2} \mathrm{~W}_{2}^{(1)}(0) \overline{\rho_{1}} \mathrm{e}^{\mathrm{i} \omega_{0}^{*} \tau_{\mathrm{k}}^{*}}+\mathrm{W}_{1}^{(1)}(0) \rho_{1} \mathrm{e}^{-\mathrm{i} \omega_{0}^{*} \tau_{\mathrm{k}}^{*}}+\frac{1}{2} \mathrm{~W}_{2}^{(2)}\left(-\tau_{\mathrm{k}}^{*}\right)+\mathrm{W}_{1}^{(2)}\left(-\tau_{\mathrm{k}}^{*}\right)\right]+\mathrm{b}_{4}\left[\mathrm{~W}_{2}^{(2)}\left(-\tau_{\mathrm{k}}^{*}\right) \overline{\rho_{1}} \mathrm{e}^{\mathrm{i} \omega_{0}^{*} \tau_{\mathrm{k}}^{*}}\right. \\
& \left.+2 W_{1}^{(2)}\left(-\tau_{k}^{*}\right) \rho_{1} e^{-i \omega_{0}^{*} \tau_{k}^{*}}\right]+b_{5}\left[\rho_{1}{ }^{2} e^{-2 i \omega_{0}^{*} \tau_{k}^{*}}+2 \rho_{1} \overline{\rho_{1}}+3 b_{6} \rho_{1}{ }^{2} \overline{\rho_{1}} e^{-i \omega_{0}^{*} \tau_{k}^{*}}\right.
\end{aligned}
$$

$$
g_{21}=2 \bar{D}\left(\overline{\rho_{2}} k_{14}+k_{24}\right)
$$

We have the following theorem for the controlled model based on the conclusions in [1].

Theorem 3. For the controlled system (10), the Hopf bifurcationis determined by the parameters $\mu_{2}, T_{2}$ and $\beta_{2}$, the conclusions are summarized asfollows:

Parameter $\mu_{2}$ determines the direction of the Hopf bifurcation. If $\mu_{2}>0$, the Hopf bifurcation is supercritical, the bifurcating periodic solutions exist for $\tau>\tau_{k}^{*}$, If $\mu_{2}<0$ the Hopf bifurcation is subcritical, the bifurcating periodic solutions exist for $\tau<\tau_{k}^{*}$.

Parameter $\beta_{2}$ determines the stability of the bifurcating periodic solutions. If $\beta_{2}<0$, the bifurcating periodic solutions is stable; if $\beta_{2}>0$, the bifurcating periodic solutions is unstable.

Parameter $T_{2}$ determines the period of the bifurcating periodic solution. If $T_{2}>0$, the period increases; If $T_{2}<0$, the period decreases.

$\mu_{2} ; T_{2}$ and $\beta_{2}$ are given as follows:

$$
\left\{\begin{array}{c}
\Delta=\frac{\mathrm{i}}{2 \omega_{0}^{*}}\left\{g_{11} g_{20}-2\left|g_{11}\right|^{2}-\frac{\left|g_{02}\right|^{2}}{3}\right\}+\frac{g_{21}}{2} \\
\mu_{2}=\frac{\operatorname{Re}\{\Delta\}}{\operatorname{Re} \dot{\lambda}\left(\tau_{k}^{*}\right)} \\
T_{2}=\frac{\operatorname{Im}\{\Delta\}+\mu_{2} \operatorname{Im} \dot{\lambda}\left(\tau_{k}^{*}\right)}{\omega_{0}^{*}} \\
\beta_{2}=2 \operatorname{Re}\{\Delta\}
\end{array}\right.
$$

Proof. Detailed proof and calculation of $g_{i j}$ are shown in [1], [13].

In the following, we present numerical simulation to verify the analytic results and the control effects of algorithm. We use the same parameters as those in [13]: $\mathrm{a}=0.8 ; \mathrm{b}=1120 ; \mathrm{c}=$ 50000 and $\mathrm{d}=0.05$, then we have:
Table 1. Numerical simulation of uncontrol system.

\begin{tabular}{ll}
\hline uncontrol system & \\
\hline$\omega_{0}$ & 16.32463278151223 \\
$\tau_{0}$ & 0.07241795255088493 \\
\hline
\end{tabular}

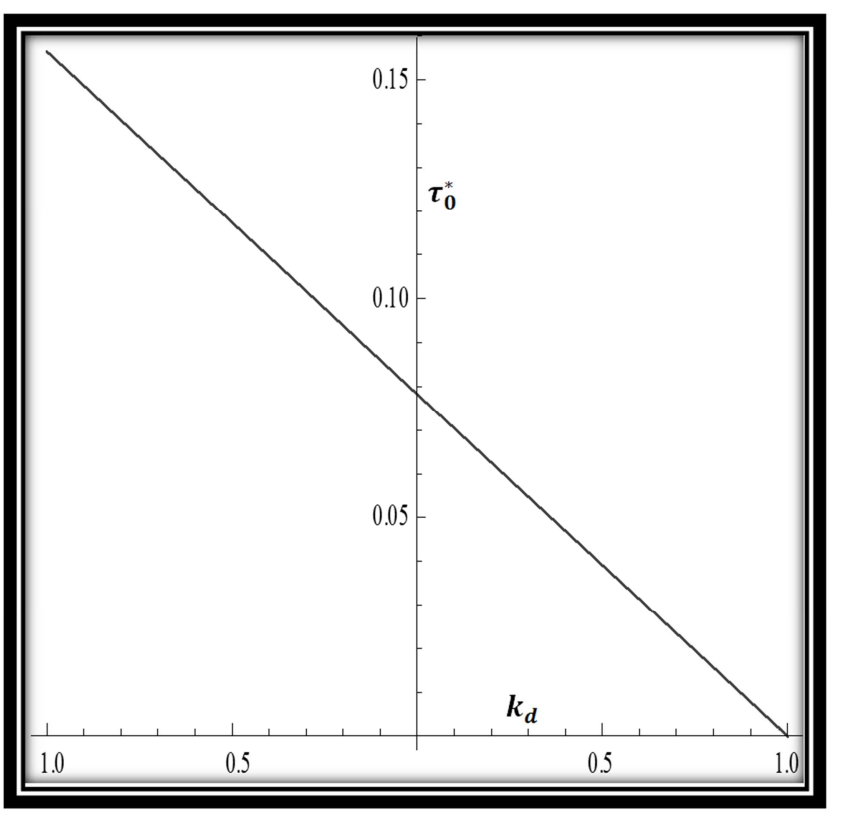

Figure 1. $\tau_{0}^{*}$ dependence on $k_{d}$ with $k_{p}=0.9$.

Table 2. Numerical simulation of control system.

\begin{tabular}{lll}
\hline control system & & \\
\hline$\omega_{0}^{*}$ & 15.483689051929085 & 27.20616359747526 \\
$\tau_{0}^{*}$ & 0.07825427250568502 & 0.043454498972068996 \\
\hline
\end{tabular}

Now we verify the performance of the controlled model with PD controller. The comparison tables will be determined by choosing $k_{p}=0.9, k_{d}=0.00003$, the onset of Hopf 
bifurcation delayed and advanced via choosing $k_{p}=$ $0.001, k_{d}=0.4$.

From figure 1, we can see, by decreasing $k_{d}, \tau_{0}^{*}$ increases. The dynamical behaviors of the uncontrolled and controlled model are illustrated in figure 2-5. Figure 2, 4 shows the system (2) in $\tau=0.07241795255088493$ and $\tau=0.07$. In order to compare controlled system with the uncontrolled system, we choose the parameters of Figure 3, 5 same as shown in Figure 2, 4. From Figure 2, 3 and tables we know the bifurcation value of uncontrolled system is $\tau_{0}=$ 0.07241795255088493 but in the controlled system is $\tau_{0}^{*}=$ 0.07825427250568502 , that shows the onset of Hopf bifurcation has been delayed obviously. Results show that the value of equilibrium point of controlled system is equal to that of uncontrolled model, which confirms that the equilibrium does not change under PD controller.
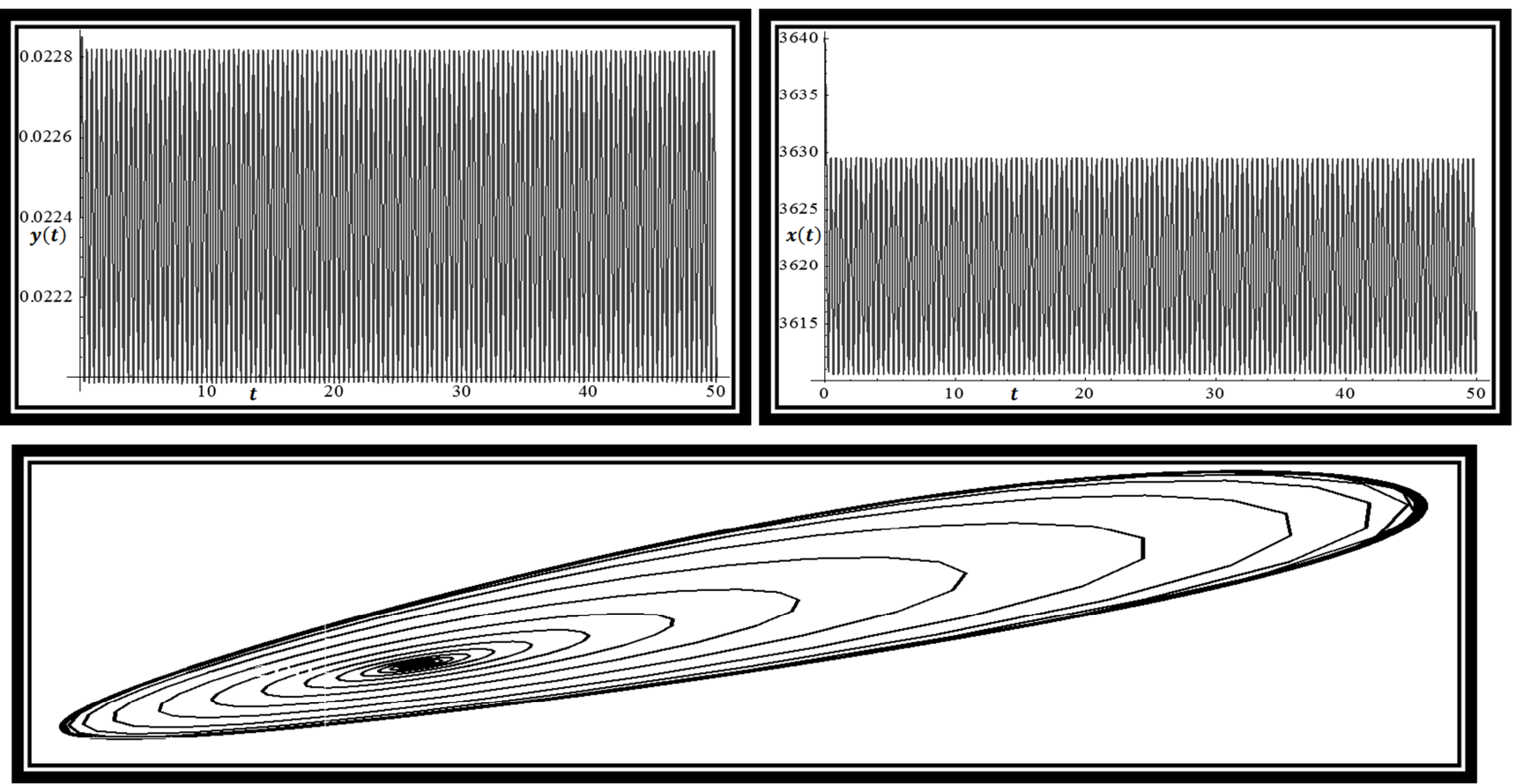

Figure 2. Waveform plot and phase plot of uncontrolled system (2) with $\tau=0.07241795255088493$.
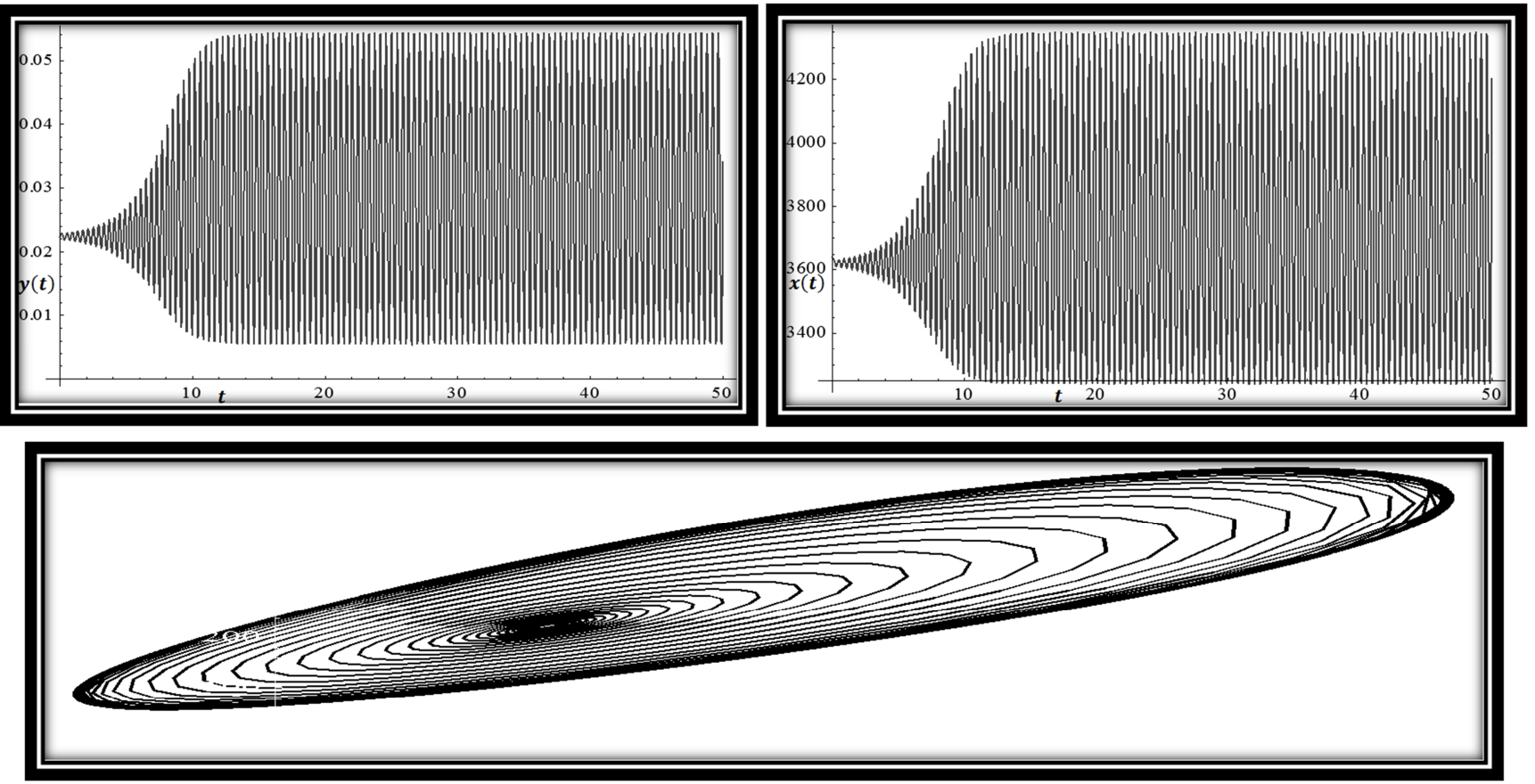

Figure 3. Waveform plot and phase plot of controlled system (10) with $\tau=0.07241795255088493, k_{p}=0.9$ and $k_{d}=0.00003$. 

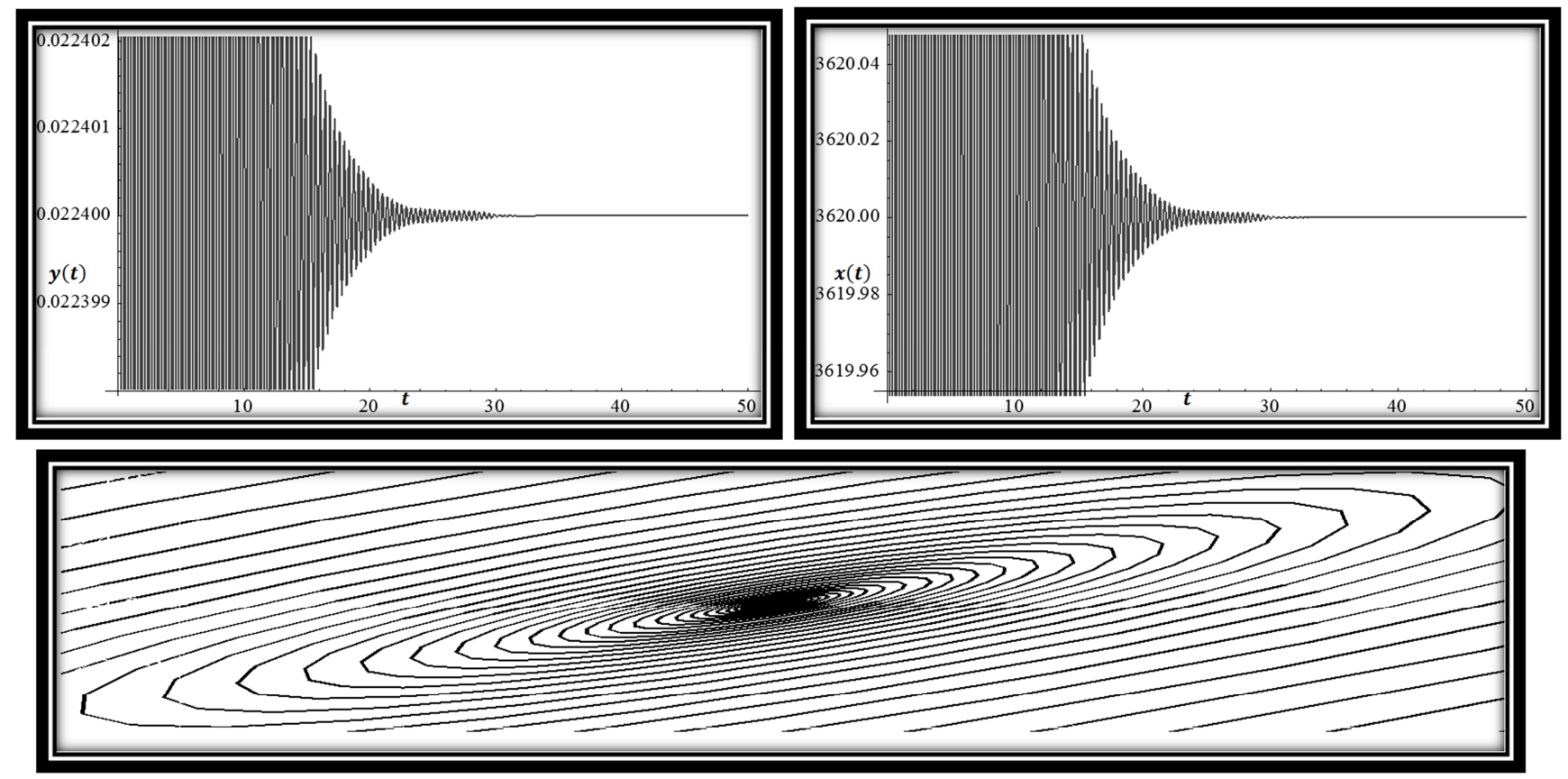

Figure 4. Waveform plot and phase plot of uncontrolled system (2) with $\tau=0.07$.
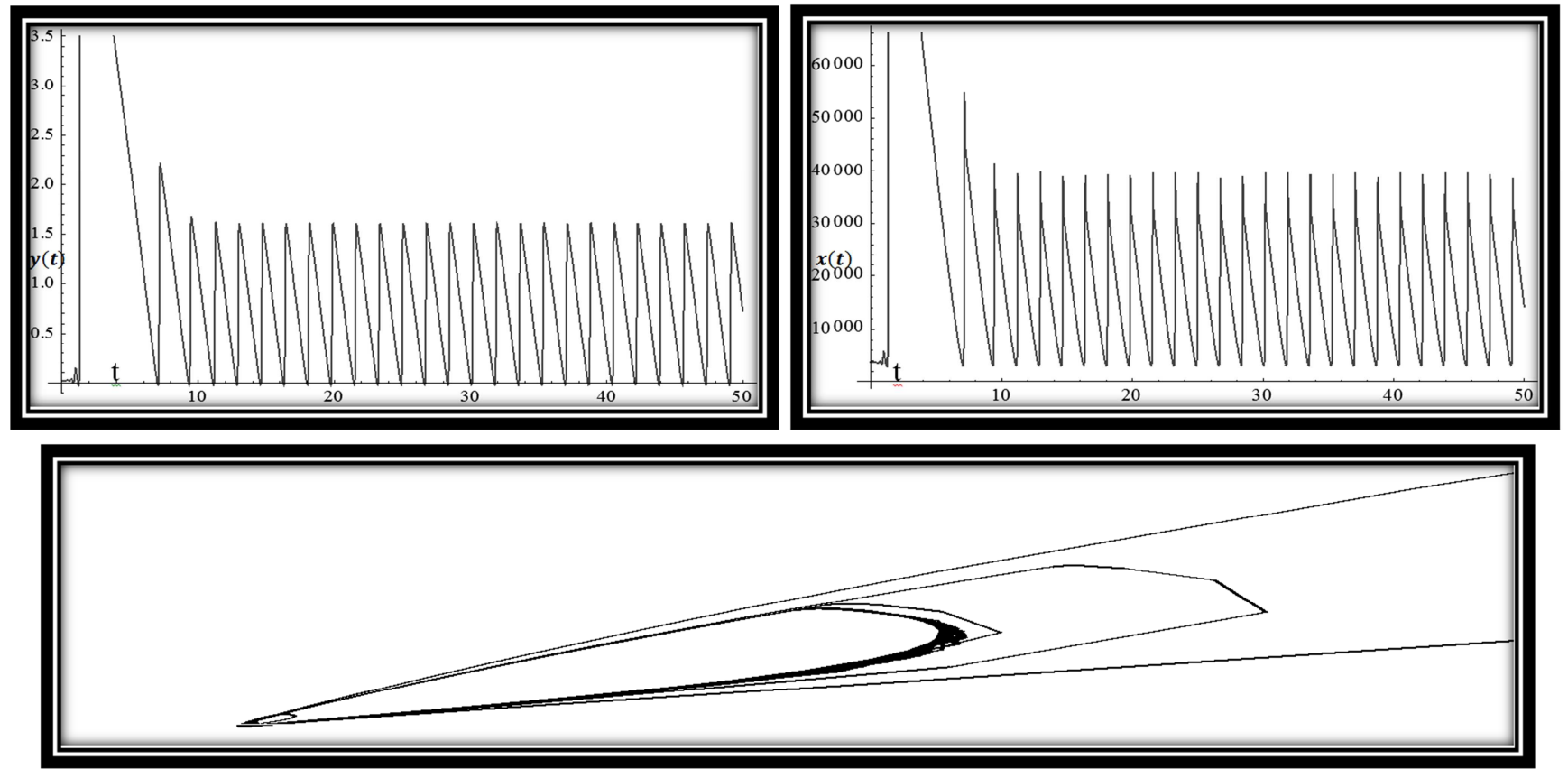

Figure 5. Waveform plot and phase plot of controlled system (10) with $\tau=0.07, k_{p}=0.0001$ and $k_{d}=0.4$.

Criterion function and Boundary constraint of the control model is

$$
\left\{\begin{aligned}
\operatorname{Max} \tau_{0}^{*}= & \frac{1}{\omega_{0}^{*}} \operatorname{Arcsin}\left(\frac{\mathrm{P}\left(\mathrm{k}_{\mathrm{p}}, \mathrm{k}_{\mathrm{d}}\right)}{\mathrm{Q}\left(\mathrm{k}_{\mathrm{p}}, \mathrm{k}_{\mathrm{d}}\right)}\right) \\
\mathrm{k}_{\mathrm{p}} & \in(-1,1) \\
\mathrm{k}_{\mathrm{d}} & \in(-1,1)
\end{aligned}\right.
$$

By proper selection of the parameters $\left(k_{p}=0.999991, k_{d}=-0.999998\right)$. We obtained $\operatorname{Max} \tau_{0}^{*}=0.157867$ and this is emphasized, by the appropriate controller the Hopf bifurcation is delayed. 


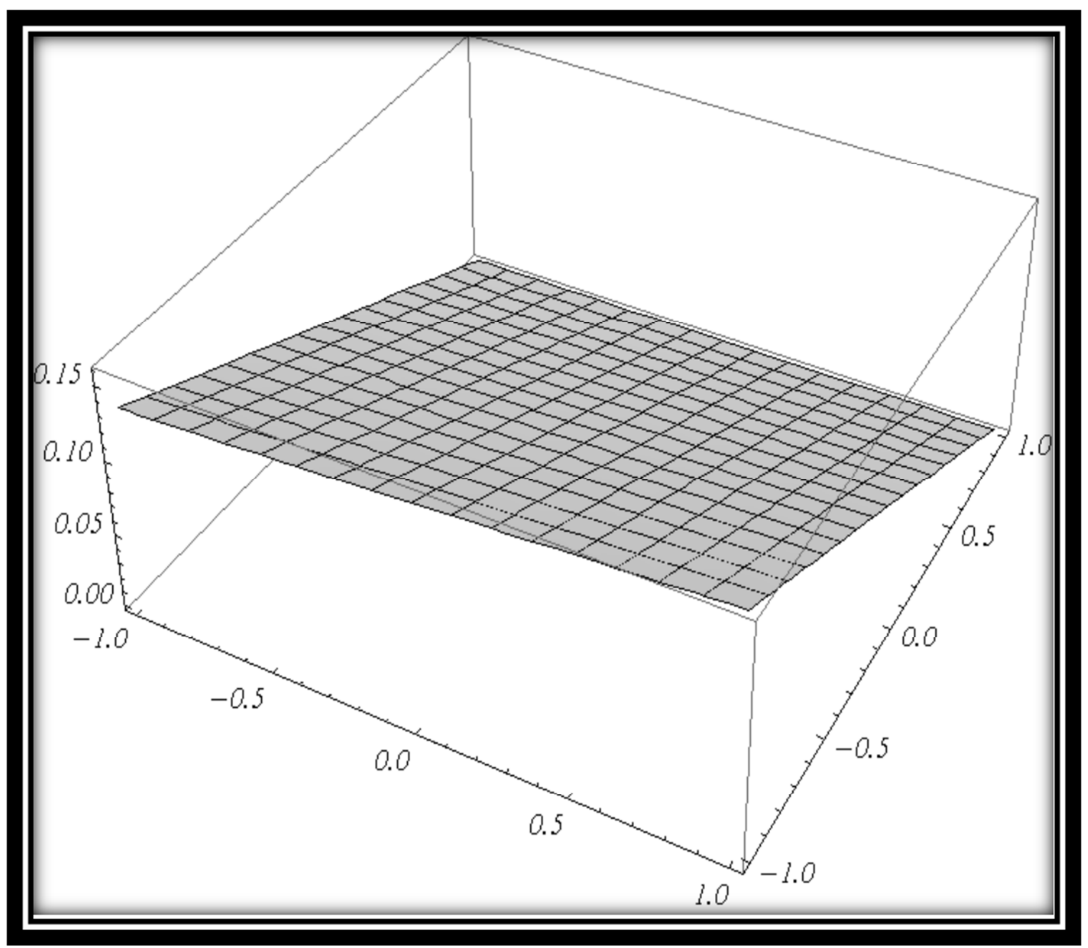

Figure 6. $\tau_{0}^{*}$ dependence on $k_{p}$ and $k_{d}$.

\section{Conclusions}

In this paper, the problem of Hopf bifurcation control for an FAST TCP model with RED gateway was studied. In order to control the Hopf bifurcation, a PD controller is applied to the model. This PD controller can successfully delay or advance the onset of an inherent bifurcation. The end theoren helped to improve model.

\section{References}

[1] B. D. Hassard, N. D. Kazarinoff, Y. H. Wan, "Theory and Applications of Hopf Bifurcation," Cambridge University Press, Cambridge, 1981.

[2] D. Ding, J. Zhu, X. Luo, and Y. Liu, "Delay induced Hopf bifurcation in a dual model of Internet congestion control algorithm," Nonlinear Analysis: Real World Applications $10.2009,2873-2883$

[3] D. Ding, X. Zhang, J. Cao, N. Wang, D. Liang, "Bifurcation control of complex networks model via PD controller," Neurocomputing 175. 2016, 1-9.

[4] Al-Bahadili, H. "Simulation in computer network design and modeling: Use and analysis", Hershey, PA: IGI Global. pp. 282, 2012.

[5] C. Jin, D. X. Wei, and S. H. Low, "BFAST TCP: Motivation, architecture, algorithms, and performance," in Proc. IEEE INFOCOM, Mar. 2004.
[6] F. Zhao, J. Zhou, N. Lu, and M. Zhang, "Stability analysis of FAST TCP based on Lyapunov function," in Proceedings of the 7th World Congress on Intelligent Control and Automation (WCICA 08). 2008, pp: 2136-2140.

[7] H. Zhang and B. Liu, "Stability of FAST TCP with time-delay in single-link multi-source networks, " in Proceedings of the IEEE International Conference on Information Networking and Automation (ICINA 10). 2010 V2-259-V2-263.

[8] J. Wang, D. X. Wei, J.-Y. Choi, and S. H. Low, "Modelling and stability of FAST TCP," in Wireless Communications 143. 2007 331-356.

[9] J.-Y. Choi, H. Kim, and S. Kwon, "Global asymptotic stability analysis of fast tcp using nonlinear small gain theorem," IEEE Communications Letters 18. 2014, 523-526.

[10] J.-Y. Choi, S.-Y. Kim, and J.-W. Kim, "Parameter condition for global asymptotic stability of FAST TCP in the presence of cross traffics," IEEE Communications Letters 14. 2010, 584-586.

[11] L. Feng, X. Gaoxiang, G. Zhi-Hong et al, "Stability analysis and control Hopf bifurcation in a FAST TCP model," in Proceedings of the 32nd IEEE Chinese Control Conference (CCC13). 2013, 1076-1080.

[12] M. Xiao, W. X. Zheng, and J. Cao, "Bifurcation control of a congestion control model via state feedback," International Journal of Bifurcation and Chaos in Applied Sciences and Engineering 23. 31, 2013.

[13] Z.-Q. Zhan, J. Zhu, and W. Li, "Stability and bifurcation analysis in a FAST TCP model with feedback delay," Nonlinear Dynamics 70. 2012, 255-267. 